\title{
Better care needed for adults in care homes
}

UK dentists have called on governments to fund dental services for residents in care homes, which are often either poor or non-existent.

The annual gathering of dentists from local dental committees (LDCs), held in Belfast (7-8 June 2018), has said that commissioning dental services for vulnerable older people is rarely a priority and it's time that this shameful situation is reversed.

Dentists have said that access to dental treatments should be given the same priority as general health for all adults in care homes, as the National Institute for Health and Care Excellence (NICE) recommended two years ago but has yet to be delivered.

Research by the British Dental Association found that less than two percent of the NHS dental budget is spent on treating these patients. It has also found where oral healthcare provision is poor that residents can experience difficulties in eating, exacerbated by untreated mouth infections or ill-fitting dentures, which can lead to dehydration and malnutrition.

Recent reports from Healthwatch inspections have found many homes' staff were 'rushed off their feet', with many residents

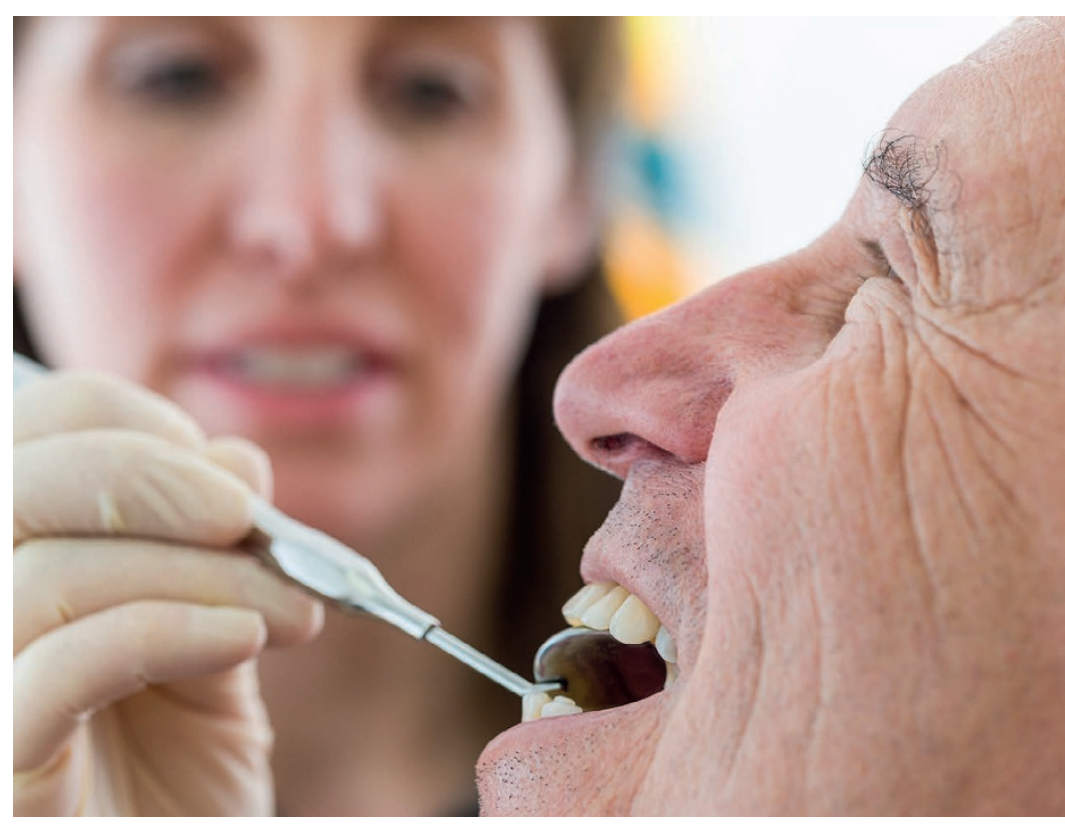
lacking access to even basic oral healthcare. It found vulnerable residents with dentures that have never been taken out, and home managers who admitted oral health was not a major priority.

Senior national adviser for the Care Quality Commission, John Milne, told delegates that when the regulator inspects care homes, it expects them to o be following the NICE guidance. He warned that the CQC is actively looking into what more it can do to improve the oral health of such residents.

\section{BOOK REVIEW}

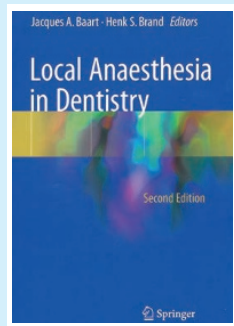

\section{LOCAL ANAESTHESIA IN DENTISTRY, SECOND EDITION}

Jacques A. Baart, Henk S. Brand

Springer; 2017

price $£ 109.99$ pp. 204

ISBN: 978-3-319-43704-0

Local anaesthesia in dentistry is a comprehensive source aimed at undergraduates, new to the techniques of local anaesthesia. Furthermore, it would be a welcome addition to the bookshelf of any qualified dentist, keen to refresh their knowledge or to use it as reference - especially on those odd days when the second inferior dental block is still not successful!

Initially published in Dutch and later translated into English, this second edition provides 13 well-versed chapters ranging from pain physiology, nerve anatomy and pharmacology, to common and more advanced techniques to achieve anaesthesia prior to dental treatment. It also delivers clinically valuable content on complications associated with the delivery of local anaesthetics and the appropriate post-operative management. The thorough chapter on 'at risk' patients also brings the importance of medical history taking and the patient-anaesthetic selection to the forefront of the reader's mind.

It also contains an important chapter on the legalities surrounding local anaesthetics - an important consideration in today's ever growing litigious society and in the aftermath of the Montgomery case. Discussing previous cases taken to court or disciplinary boards the world over, it picks apart the issues and delivers solemn advice for practitioners undertaking local anaesthetics.

Throughout the book, the illustrations support the subject matter vividly. It demonstrates the techniques through a combination of anatomical diagrams, skull models and intra-oral patient photography. This brings the techniques to life for the reader, providing a step-by-step guide to achieving successful anaesthesia.

Within each chapter, there are also helpful content boxes which highlight key points and deliver practical insights from the authors' own experiences with anaesthetics, thus creating a personalised and reassuring approach to the teaching of local anaesthetics.

Those who read this book should be aware of the dosage differences between those published here and what is available for use in the United Kingdom. For example, calculations and recommendations within the book are based on $1.8 \mathrm{ml}$ cartridges and not the $2.2 \mathrm{ml}$ cartridges used in UK practices; however, the basic principle of dose calculation remains the same.

Local anaesthesia in dentistry is an excellent and worthwhile source for dental students preparing to embark on their first local anaesthetic injections. It has a succinct and rational lay out, supplying the reader with appropriate information and handy tips. It builds from the basic foundations of pain physiology up to the correct positioning of operator and patient to deliver a successful inferior dental nerve block. As mentioned before, even those long qualified may benefit from this read, retaining it as a text for reference, especially in those challenging cases resistant to anaesthesia.

Fiona Wright 